\title{
Diagramas de manejo de densidad para renovales de roble, raulí y coigüe en Chile
}

\author{
Stocking management charts for roble, raulí and coigüe second growth forests in Chile
}

\author{
Salvador A Gezana*, Alicia Ortegab, Ernesto Andenmattenc \\ *Autor de correspondencia: aRothamsted Research, Harpenden, Hertfordshire AL5 2JQ, United Kingdom, Tel.: 44(0)1582-763- \\ 133, fax: 44(0)1582-467-116, salvador.gezan@bbsrc.ac.uk \\ bUniversidad Austral de Chile, Facultad de Ciencias Forestales, Valdivia, Chile. \\ cInstituto Nacional de Tecnología Agropecuaria, Campo Forestal Gral. San Martín, Argentina.
}

\begin{abstract}
SUMMARY
A total of 214 permanent and temporary plots from roble (Nothofagus obliqua), raulí (N. alpina) and coigüe (N. dombeyi) pure and mixed second growth forests located between $37^{\circ} 30^{\prime}$ and $42^{\circ} 00^{\prime}$ south latitude in Chile were used to fit an average size-density model. This model was then modified to obtain maximum density curves, and to construct stocking management charts with the aim to support planning of thinning, and to help the application of CONAF's second growth forests management guidelines. The final size-density model considered a common slope and specific intercepts for three different compositions: raulí mixed, roble and coigüe. Comparisons of these models with others reported in the literature showed an acceptable level of similarity. Concerning load site capacity, the lowest levels were found for the composition roble, and the highest for coigüe. The following reference lines are suggested for the use of the presented stocking charts: 100\% (line A or maximum density), $60 \%$ (line B or beginning of self-thinning), 30\% (line C or load site capacity inferior limit), and 20\% (line D or minimum density). Additionally, applications and methodology for the construction of these stocking charts are fully described together with concepts related to each of the reference lines.
\end{abstract}

Key words: thinning, size-density, Nothofagus, self-thinning, SDI.

\section{RESUMEN}

Un total de 214 parcelas permanentes y temporales de renovales puros o mixtos de roble (Nothofagus obliqua), raulí (N. alpina) y coigüe ( $N$. dombeyi) localizadas entre los $37^{\circ} 30^{\prime}$ y $42^{\circ} 00^{\prime}$ de latitud sur en Chile fueron usadas para obtener un modelo de tamañodensidad. Este modelo fue posteriormente modificado para obtener curvas de densidad máxima y para construir diagramas de manejo de densidad con el objetivo de apoyar la planificación de raleos, facilitar la aplicación de las normas de manejo de renovales de la Corporación Nacional Forestal, CONAF. El modelo final de tamaño-densidad consideró una pendiente común e interceptos específicos para tres diferentes composiciones: raulí mixto, roble y coigüe. La comparación de estos modelos con otros reportados por la literatura indicó un grado aceptable de similitud. En relación con la capacidad de carga de uso del sitio se encontraron los niveles más bajos para la composición roble y los niveles más altos para coigüe. Se sugiere considerar las siguientes líneas de referencia para el uso adecuado de los diagramas propuestos: 100\% (línea A o de densidad máxima), 60\% (línea B o de inicio del autorraleo), 30\% (línea C o límite inferior de ocupación del sitio) y 20\% (línea D o nivel mínimo de densidad). Adicionalmente, el uso y metodología para la obtención de estos diagramas son descritos en detalle junto con conceptos relacionados a cada una de estas líneas de referencia.

Palabras clave: raleo, tamaño-densidad, Nothofagus, autorraleo, IDR.

\section{INTRODUCCIÓN}

Los renovales de roble (Nothofagus obliqua (Mirb.) Oerst.), raulí (Nothofagus alpina (Poepp. et Endl.) Oerst.) y coigüe (Nothofagus alpina (Mirb.) Oerst.) constituyen uno de los bosques naturales más interesantes de Chile. De acuerdo al último catastro de bosque nativo existen alrededor de 1,2 millones de hectáreas de renovales del tipo forestal roble-raulí-coigüe sólo en Chile (CONAF et al. 1999). Esta amplia superficie, el valor de la madera de sus especies y sus tasas de crecimiento (Donoso et al. 1993, Echeverría y Lara 2004) hacen que este recurso represente una reserva de alto potencial económico dentro del sector forestal chileno. Los bosques naturales de este tipo forestal son uno de los más estudiados. La principal intervención silvicultural evaluada es el raleo, mediante el cual se controla la densidad del rodal para concentrar el crecimiento en los mejores individuos remanentes y así 
acortar el periodo de rotación (Lara et al. 1998, Martínez 1998). A nivel general, para cada raleo se recomienda rebajar el área basal original entre un 30 y $40 \%$, con intensidades mayores para rodales jóvenes y a intervalos de entre 10-12 años, afectando principalmente los estratos intermedios y superiores (Grosse y Quiroz 1998).

$\mathrm{La}$ alta variabilidad existente en composición y estructura de estos renovales a lo largo de su distribución geográfica (Lara et al. 1998) se debe, entre otras cosas, a diversas calidades de sitio, grados de alteración antrópica, composiciones florísticas y/o variables edafoclimáticas. Esta variabilidad se traduce en diferentes tasas de crecimiento, dificultando la aplicación de una receta única de raleo, requiriendo que la intensidad, tipo y periodicidad de éstos sea planificada de acuerdo a cada situación particular y a los objetivos de manejo deseados (Grosse y Quiroz 1998).

Un gran avance en este sentido es la publicación y aplicación por parte de CONAF de las normas de manejo para renovales de roble, raulí y coigüe. Ellas entregan pautas claras y generales que simplifican la aplicación de los raleos, con la ventaja adicional de que se facilita la evaluación y fiscalización de estas intervenciones (Lara et al. 1998). Estas normas caracterizan el estado actual del rodal a través de la incorporación de dos variables dasométricas claves: el diámetro cuadrático y el número de árboles por hectárea. Con el diámetro cuadrático se consultan tablas por subtipo forestal, las que determinan la densidad mínima inicial para ejecutar un raleo y la densidad mínima a dejar después de la intervención, a fin de asegurar un nivel adecuado de productividad, protección del sitio y otras funciones del bosque. Además, se restringe la intervención a una extracción máxima del área basal de un 35 y $40 \%$ para renovales de coigüe y roble-raulí, respectivamente.

Las características de estas normas en relación a sus requerimientos de información son similares a las de los "diagramas de manejo de densidad" o "guías de densidad" originalmente descritas por Gingrich (1967). Estos diagramas describen gráficamente la relación existente entre el área basal, el número de árboles por hectárea y el diámetro cuadrático, e incluyen una serie de curvas de referencia para diversos niveles de ocupación del sitio. Su uso permite describir la situación actual de un rodal y compararlo con condiciones predeterminadas de acuerdo a objetivos de manejo previamente especificados (Rogers 1983). Por esto se les considera una valiosa herramienta que facilita la adecuada planificación y monitoreo de intervenciones silvícolas, maximizando la utilización del sitio mediante un control adecuado de los niveles de competencia del rodal.

Los diagramas están construidos sobre la base de la relación lineal (en escala logarítmica) de tamaño-densidad o ley de autorraleo que se considera independiente de la edad y calidad de sitio del rodal (Reineke 1933, Long 1985). Por este motivo, los diagramas pueden ser aplicables en diversas condiciones ambientales. Sin embargo,
Zeide (1987) indica que la edad, la calidad del sitio y otros factores pueden afectar esta relación lineal. Por ejemplo, Zeide (1995) sostiene que con el tiempo los árboles pierden su capacidad para ocupar espacios dejados por árboles muertos y, por lo tanto, la ocupación máxima del sitio disminuye y el uso de una relación cuadrática es más adecuado. No obstante, bajo rangos normales de desarrollo, como son las condiciones muestreadas en este estudio, se espera que esta relación lineal sea correcta.

Los diagramas de manejo de densidad han sido previamente desarrollados para varias especies de coníferas y latifoliadas del hemisferio norte (Gingrich 1967, Rogers 1983, Wittwer et al. 1998, Day 1998). Chauchard et al. (2001) presentan un diagrama para rodales puros y mixtos de roble, raulí y coigüe, el que está basado en mediciones de renovales adultos de Argentina. Es posible que estos rodales representen una situación diferente a la encontrada en Chile debido a que se encuentran localizados sobre los 600 m s.n.m. y dado que presentan diámetros cuadráticos superiores $(30$ a $80 \mathrm{~cm})$ a los encontrados en el país. Incluso, Donoso et al. (2004) indican que los rodales de roble de Argentina, producto de su distribución discontinua, podrían eventualmente ser considerados una variedad ecotípica diferente.

Los objetivos del presente estudio consisten en: a) presentar diagramas de manejo de densidad para renovales de roble, raulí y coigüe localizados en Chile, b) proponer niveles de densidad de referencia para apoyar la planificación de raleos para estos renovales, y c) describir en detalle la metodología para la obtención de estos diagramas, para fomentar su uso y generación a futuro para otros tipos forestales.

\section{MÉTODOS}

Fuente de información. Se utilizó una red de parcelas permanentes y otra de parcelas temporales de superficies entre 250 y $500 \mathrm{~m}^{2}$ (214 parcelas en total, cuadro 1). Se consideraron parcelas de $250 \mathrm{~m}^{2}$ sólo para aquellas situaciones en donde la densidad fuese superior a 4.800 árboles por hectárea. Para cada parcela se midieron todos los árboles con DAP $>5 \mathrm{~cm}$ y adicionalmente se eligió una submuestra proporcional a la distribución diamétrica de alrededor de 15 árboles (parcelas permanentes) o seis árboles (parcelas temporales), a los cuales se les midió la altura total y se les determinó la edad mediante la obtención de un tarugo de crecimiento o una rodela. La altura dominante fue calculada como el promedio de los 100 árboles más gruesos por hectárea. Todas las parcelas fueron establecidas en bosques puros o mixtos de segundo crecimiento de roble, raulí y coigüe localizados entre los $37^{\circ} 30^{\prime}$ y $42^{\circ} 00^{\prime}$ de latitud sur en Chile, los cuales se caracterizaron por tener nulos o bajos niveles de intervención antrópica. La elección de los puntos de muestreo se hizo en forma proporcional a la caracterización de estos bosques 
Cuadro 1. Estadísticos descriptivos de las parcelas utilizadas. NHA: número de árboles por hectárea, DC: diámetro cuadrático, $\mathrm{HD}$ : altura dominante, $\mathrm{AB}$ : área basal, $\mathrm{N}$ : número de observaciones.

Descriptive statistics of available plots. NHA: trees per hectare, DC: quadratic diameter, HD: dominant height, $\mathrm{AB}$ basal area, $\mathrm{N}$ : number of observations.

\begin{tabular}{lcccc}
\hline Parámetro & N & Mínimo & Media \pm S & Máximo \\
\hline NHA (árboles/ha) & 26 & 490 & $2.321 \pm 1.184$ & 4.880 \\
DC (cm) & 26 & 10,5 & $17,6 \pm 5,7$ & 38,5 \\
HD (m) & 25 & 15,6 & $22,5 \pm 5,2$ & 35,1 \\
AB (m²/ha) & 26 & 26,0 & $47,5 \pm 12,8$ & 72,3 \\
\hline \multicolumn{5}{c}{ Roble } \\
\hline NHA (árboles/ha) & 115 & 460 & $2.177 \pm 1.218$ & 5.080 \\
DC (cm) & 115 & 6,8 & $16,5 \pm 6,4$ & 34,7 \\
HD (m) & 111 & 10,0 & $22,9 \pm 7,1$ & 42,4 \\
AB (m²/ha) & 115 & 14,5 & $36,1 \pm 11,4$ & 62,6 \\
\hline \multicolumn{5}{c}{ Coigüe } \\
NHA (árboles/ha) & 47 & 880 & $2.674 \pm 1.288$ & 5.560 \\
DC (cm) & 47 & 9,2 & $17,7 \pm 5,4$ & 30,5 \\
HD (m) & 45 & 12,4 & $22,0 \pm 5,8$ & 37,7 \\
AB (m²/ha) & 47 & 25,1 & $55,8 \pm 17,0$ & 98,4 \\
\hline & \multicolumn{5}{c}{ Mixto } \\
\hline NHA (árboles/ha) & 26 & 784 & $2.726 \pm 1.217$ & 5.600 \\
DC (cm) & 26 & 8,4 & $15,6 \pm 4,6$ & 24,5 \\
HD (m) & 26 & 9,9 & $20,5 \pm 4,9$ & 28,5 \\
AB (m²/ha) & 26 & 17,2 & $45,4 \pm 13,9$ & 78,1 \\
\hline & \multicolumn{5}{c}{} \\
\hline
\end{tabular}

de acuerdo con CONAF et al. (1999), considerando los siguientes estratos: composición, altura del dosel dominante, cobertura y distribución geográfica.

Las parcelas seleccionadas correspondieron a aquellas en donde las especies de Nothofagus (roble, raulí y coigüe) presentaban un área basal superior al 60\% del área basal total. Para cada parcela se calculó una composición dominante de acuerdo a la especie que presentaba al menos un $70 \%$ del área basal total de las tres especies de Nothofagus. La composición se clasificó como mixta para aquellas parcelas en donde ninguna de las especies dominaba. Algunas parcelas fueron eliminadas del análisis debido a que no se encontraban en niveles altos de ocupación del sitio, las que fueron identificadas como observaciones alejadas de la nube de puntos obtenida a partir de la relación número de árboles versus diámetro cuadrático.

Modelo de tamaño-densidad. Para poblaciones que presentan mortalidad debido a la competencia entre individuos producto de la alta densidad (aglomeración), existe una relación entre el tamaño promedio y el número de individuos que además de ser fácil de modelar es relativamente estable bajo diversas situaciones. En rodales forestales, el modelo de tamaño-densidad más difundido es el propuesto por Reineke (1933) que corresponde a una relación lineal entre el logaritmo del número de árboles y el logaritmo del diámetro cuadrático. Este modelo es:

$$
\ln (\mathrm{NHA})=\mathrm{a}+\mathrm{b} \ln (\mathrm{DC})
$$

cuya versión no linealizada es:

$$
\mathrm{NHA}=\exp (\mathrm{a}) \mathrm{DC}^{\mathrm{b}}=\mathrm{a}_{0} \mathrm{DC}^{\mathrm{b}}
$$

donde, NHA es el número total de árboles por hectárea, DC es el diámetro cuadrático $(\mathrm{cm})$, a y b son parámetros a ser estimados. Además, nótese que $\mathrm{a}_{0}=\exp (\mathrm{a})$.

Para varias especies de coníferas, Reineke (1933) postuló que el valor del parámetro de la pendiente (b) es una constante universal igual a $-1,605$. Sin embargo, diversos estudios postulan que este parámetro es específico para cada especie y debe ser estimado a partir de los datos (Río et al. 2001). Por otro lado, diferentes composiciones de especies pueden influir sobre esta relación (Roach 1977). Sin embargo, para el presente estudio se espera que este efecto sea menor debido a que sólo fueron elegidos rodales relativamente puros.

Se realizó un único ajuste considerando todas las observaciones en forma simultánea, usando la expresión linealizada de la relación de tamaño-densidad y considerando un intercepto $\left(a_{\mathrm{i}}\right)$ y una pendiente $\left(\mathrm{b}_{\mathrm{i}}\right)$ específica para cada composición. Se prefirió ajustar el modelo en su forma lineal ya que la transformación logarítmica permite controlar la heterogeneidad de varianzas. Debido al interés por agrupar composiciones, se procedió en una primera etapa a evaluar la hipótesis de que no hay diferencias significativas entre las pendientes $\left(b_{i}\right)$ entre todas las composiciones. Posteriormente, se compararon los interceptos $\left(\mathrm{a}_{\mathrm{i}}\right)$ entre todos los posibles pares de composiciones a fin de evaluar si algunos grupos se pueden combinar. Las estimaciones de parámetros y resultados de las pruebas de hipótesis se obtuvieron usando el procedimiento de modelos lineales generales PROC GLM de SAS Version 8 (SAS Institute 1999).

Curvas de densidad máxima. La curva de densidad máxima corresponde a una relación teórica que describe la trayectoria seguida por el número de individuos de aquellos rodales que se encuentran en completa ocupación del sitio, es decir, el nivel máximo de competencia, y por ende es frecuentemente utilizado como indicador del límite superior de carga del rodal.

El ajuste del modelo de Reineke (1933) describe una recta promedio de los datos. Esto implica que las curvas ajustadas pasan por el centro de la nube de datos (figura 1). Sin embargo, la curva de densidad máxima corresponde al límite superior y, en la práctica, no se encuentran rodales en densidad completa producto de la muerte de individuos debido a la competencia. Este nivel máximo se obtiene incrementando la magnitud del intercepto para estimar un 

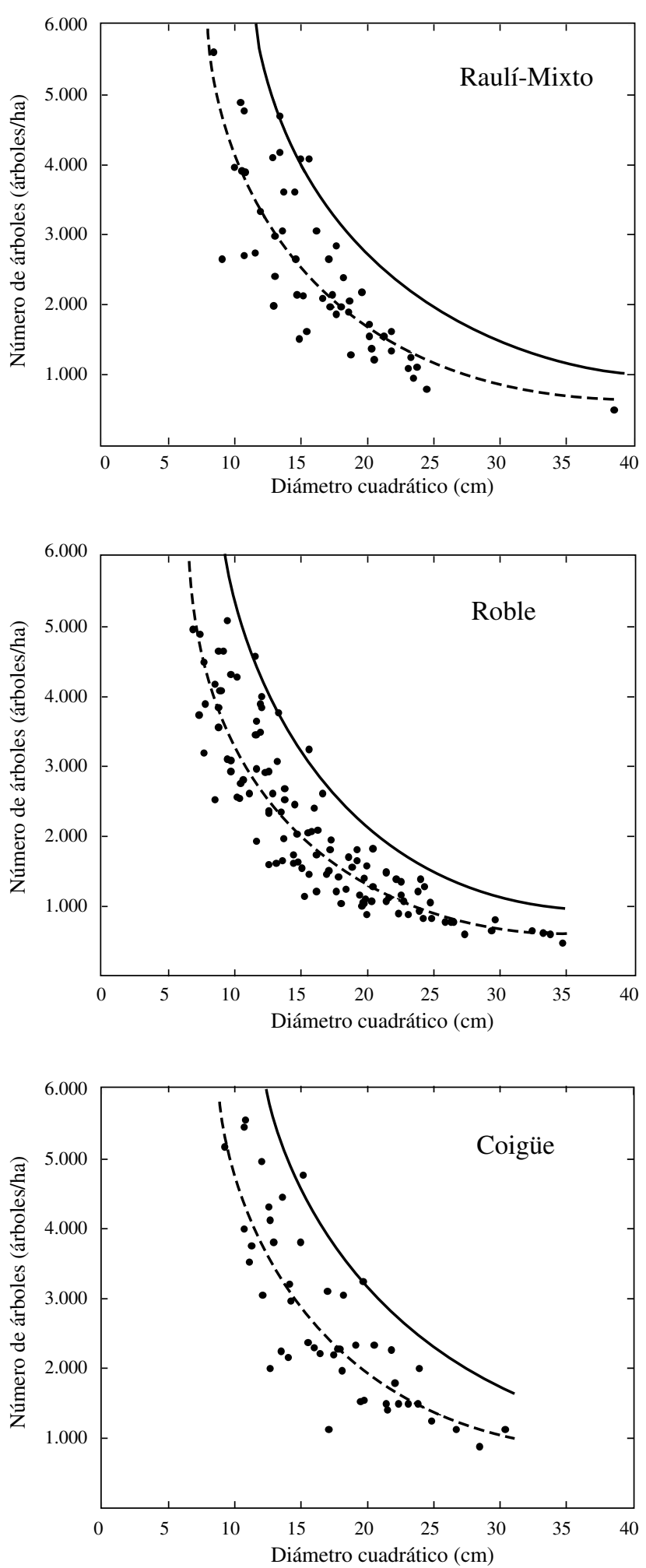

Figura 1. Ajuste del modelo de Reineke (1933) entre el número de árboles por hectárea y el diámetro cuadrático para cada una de las composiciones. Línea punteada: densidad promedio (ecuación [2]). Línea continua: densidad máxima (ecuación [3]).

Reineke's (1993) fitted model between quadratic diameter and number of trees per hectare for each composition. Dotted line: average density (equation [2]). Continuous line: maximum density (equation [4]). $\mathrm{a}_{\max }$. Diversos métodos han sido propuestos para obtener este parámetro (Wittwer et al. 1998, Day 1998). Sin embargo, de acuerdo a lo sugerido por Wittwer et al. (1998) se optó por incrementar cada intercepto $\mathrm{a}_{\mathrm{i}}$ en 1,96 desviaciones estándar del error del modelo ( $\left.\mathrm{s}^{2}\right)$, es decir, $\mathrm{a}_{\max }=1,96$ $\mathrm{x} \mathrm{a}_{\mathrm{i}}$. De este modo, asintóticamente sólo existe un $2,5 \%$ de probabilidad de encontrar parcelas que sobrepasen esta curva de densidad máxima. Así, la expresión de densidad máxima para cada composición queda:

$$
\mathrm{NHA}_{\max }=\exp \left(\mathrm{a}_{\text {max }}\right) \mathrm{DC}^{\mathrm{b}}
$$

Índice de densidad de rodal. Un indicador de utilidad que puede ser calculado a partir de la relación de tamaño-densidad es el índice de densidad de rodal (IDR) originalmente propuesto por Reineke (1933), y que permite ser utilizado como indicador del nivel actual de carga de un sitio. Este índice corresponde al número de árboles por hectárea que un rodal específico podría alcanzar si su diámetro cuadrático fuera de $25 \mathrm{~cm}$ (o 10 pulgadas).

Al igual que con la relación de tamaño-densidad, se puede obtener para cada una de las composiciones su valor máximo del índice de densidad de rodal $\left(\mathrm{IDR}_{\max }\right)$. Este indicador permite estudiar diferentes rodales (o especies) y compararlos desde un punto de vista ecofisiológico debido a que rodales que presentan valores más altos de $\mathrm{IDR}_{\max }$ frecuentemente corresponden a bosques dominados por especies tolerantes a la sombra o a mayores niveles de competencia (Wittwer et al. 1998). La siguiente expresión se utilizó para cada composición:

$$
\mathrm{IDR}_{\text {max }}=\exp \left(a_{\max }\right)(25)^{b}
$$

Curva de área basal máxima. La curva de área basal máxima corresponde a la curva de máxima ocupación del sitio que es expresada en términos de área basal a partir del número de árboles por hectárea, correspondiendo a la situación en donde el IDR es máximo. Ésta se obtiene definiendo un área basal máxima, a partir de un número máximo de árboles y un diámetro cuadrático de referencia:

$$
\mathrm{AB}_{\max }=\frac{\pi}{40.000} \mathrm{NHA}_{\max } \mathrm{DC}^{2}
$$

A fin de obtener un modelo de $\mathrm{AB}_{\max }$ que dependa únicamente del $\mathrm{NHA}_{\max }$ se puede despejar el DC de la ecuación 3, quedando:

$$
\mathrm{DC}=\left(\frac{\mathrm{NHA}_{\max }}{\exp \left(\mathrm{a}_{\max }\right)}\right) 1 / \mathrm{b}
$$

el que luego se reemplaza en la ecuación 5 y se obtiene: 


$$
\mathrm{AB}_{\max }=\frac{}{40.000} \mathrm{NHA}_{\max }\left(\frac{\mathrm{NHA}_{\text {max }}}{\exp \left(\mathrm{a}_{\max }\right)}\right)^{2 / b}
$$

Construcción de diagramas de manejo de densidad. Los diagramas están basados en la curva de $\mathrm{AB}_{\max }$, y en ellos se incluyen varias líneas paralelas como proporciones del nivel de densidad máximo del sitio. Para esto, se modifica la ecuación 7 multiplicándola por una constante p que varía entre 0 y 1 . Además, como referencia se incluyen rectas para indicar los diferentes diámetros cuadráticos.

\section{RESULTADOS}

Los resultados de las pruebas de hipótesis del modelo lineal ajustado indicaron que no existen diferencias estadísticamente significativas entre las pendientes de las diferentes composiciones $(P=0,6658)$. Por otro lado, el ajuste del modelo con una única pendiente mostró diferencias estadísticamente significativas entre los interceptos $(P<0,001)$. Sin embargo, al comparar los interceptos en forma individual para diferentes pares de composiciones, se encontró que sólo raulí y mixto no eran significativamente diferentes $(P=0,8780)$. Por lo tanto, se optó por unir estos grupos y obtener un intercepto común para ellos. Finalmente, la prueba de hipótesis de que la pendiente es igual al valor propuesto por Reineke (1933) de $-1,605$ para todas las composiciones fue rechazada $(P<0.0001)$, confirmando que la pendiente es un parámetro específico para cada especie.

El modelo final presentó un coeficiente de determinación $\left(\mathrm{R}^{2}\right)$ de 0,8025 y un error estándar $\left(\mathrm{s}^{2}\right)$ de 0,0636 . Las estimaciones de los parámetros junto con sus errores estándar y los cálculos de valores máximos del intercepto $\mathrm{a}_{\max }$ se detallan en el cuadro 2. Por otro lado, los valores de $\mathrm{IDR}_{\max }$ obtenidos fueron de 1.933,9, 1.521,7 y 2.238,6 para las composiciones raulí mixto, roble y coigüe, respectivamente.

Las relaciones de densidad máxima para cada una de las composiciones finales (raulí mixto, roble y coigüe) muestran, como es de esperar, que un número reducido de parcelas se encuentra sobre las curvas de densidad máxima (figura 1). Estos gráficos junto con las respectivas

Cuadro 2. Parámetros (error estándar entre paréntesis) y número de observaciones $(\mathrm{N})$.

Parameters (standard error in parenthesis) and number of observations $(\mathrm{N})$.

\begin{tabular}{lcccr}
\hline Composición & Parámetro & Estimación & $\begin{array}{c}\text { Intercepto } \\
\text { máximo }\end{array}$ & $\mathrm{N}$ \\
\hline Raulí-mixto & $\mathrm{a}_{1}$ & $11,6167(0,1374)$ & 12,1099 & 52 \\
Roble & $\mathrm{a}_{2}$ & $11,3770(0,1448)$ & 11,8702 & 115 \\
Coigüe & $\mathrm{a}_{3}$ & $11,7630(0,1415)$ & 12,2562 & 47 \\
Todos & $\mathrm{b}$ & $-1,4112(0,0496)$ & - & 214 \\
\hline
\end{tabular}

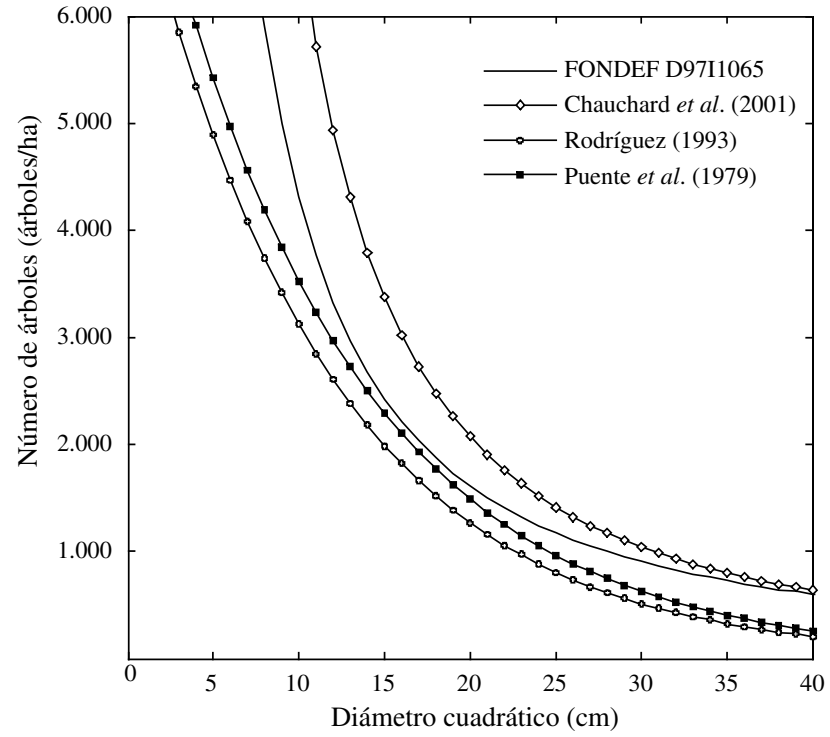

Figura 2. Comparación de diversas relaciones tamaño-densidad para rodales mixtos de roble-raulí-coigüe o roble-raulí. Nota: "FONDEF D97I1065" corresponde al presente estudio.

Comparison of several size-density relationships for mixed stands of roble-raulí-coigüe or roble-raulí. Note: "FONDEF D97I1065" corresponds to the present study.

ecuaciones pueden ser usados para predecir los niveles de densidad máximos esperados a un diámetro cuadrático específico, permitiendo además calcular índices para determinar el nivel de ocupación actual del sitio para un rodal particular. Adicionalmente, en la figura 2 se compara la relación tamaño-densidad obtenida por este estudio para la composición raulí mixto en relación a modelos reportados en la literatura. Finalmente, en la figura 3 se muestran los diagramas de manejo de densidad para cada una de las composiciones; en ellas se incorporaron además líneas que identifican diversos niveles de capacidad de carga.

\section{DISCUSIÓN}

Para rodales del género Nothofagus se han reportado anteriormente resultados del ajuste de diversos modelos de tamaño-densidad. Puente et al. (1979) y Rodríguez (1993) presentan resultados para renovales de roble-raulí en ensayos localizados entre Malleco y Valdivia, usando un modelo lineal de tamaño-densidad que relaciona el diámetro cuadrático sin transformar con el logaritmo natural del número de árboles. Chauchard et al. (1999) ajustaron un modelo idéntico a la ecuación 1 para rodales puros y mixtos de Nothofagus spp. en Argentina. Sin embargo, su base de datos considera diámetros cuadráticos de rodales adultos (de 30 a $80 \mathrm{~cm}$ ). Al comparar estos modelos junto con el obtenido por este estudio para la composición raulí mixto (figura 2), se observa que todos 
ellos son relativamente similares, presentando diferencias mayores en los diámetros extremos y, además, el modelo promedio ajustado en este estudio se encuentra al centro de los otros modelos.
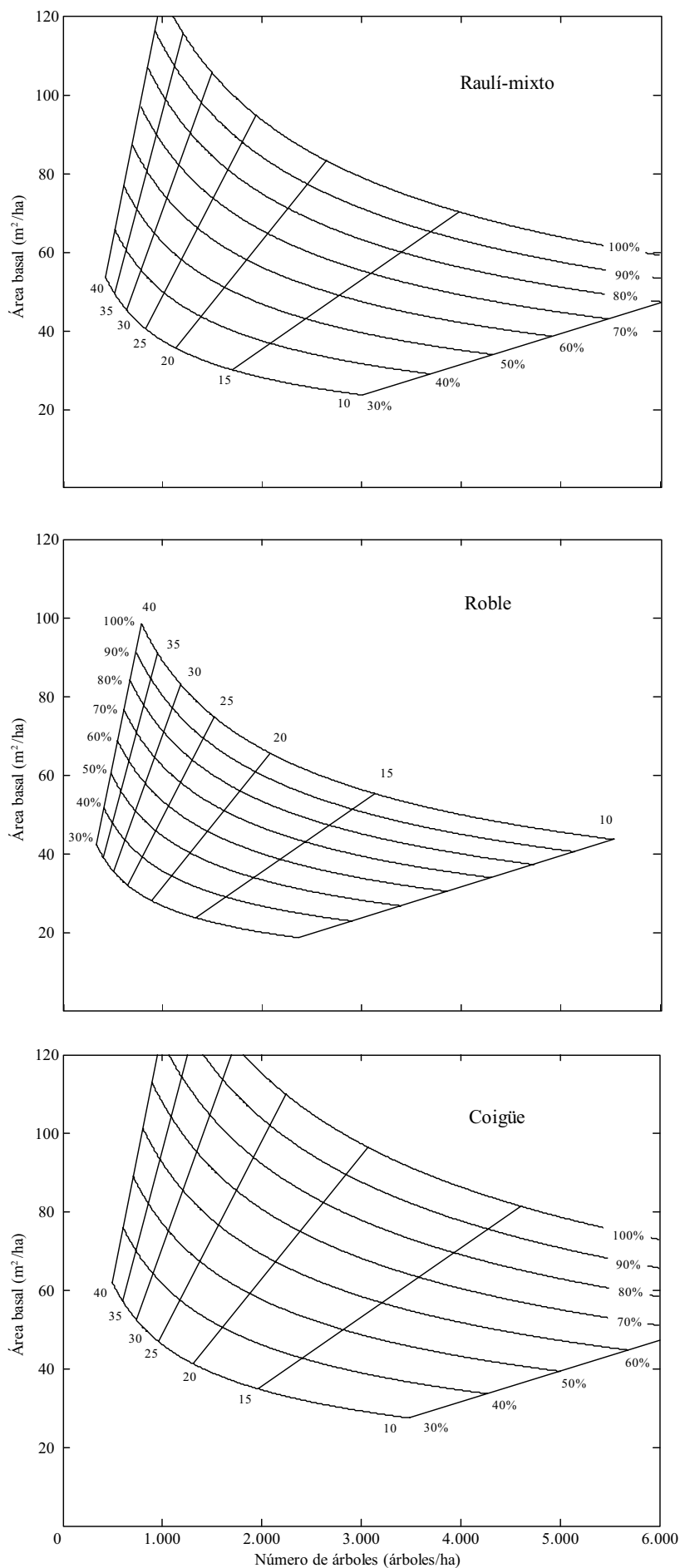

Figura 3. Diagramas de manejo de densidad para rodales de composición raulí mixto, roble y coigüe. Líneas indican diferentes diámetros cuadráticos (verticales) y niveles de capacidad de carga (horizontales).

Stocking management chart for raulí-mixed, roble and coigüe stands. Lines indicate different quadratic diameters (vertical) and stock capacity levels (horizontal).
En relación con los parámetros estimados para el modelo lineal final, los valores más altos de $\mathrm{a}_{\max }$ fueron encontrados para coigüe, y los menores para roble. Esto indica que la capacidad de carga de los rodales dominados por coigüe es más alta, y por lo tanto presentaría una mayor tolerancia a la competencia. Las diferencias de hábitat entre las diferentes composiciones podrían explicar en alguna medida este fenómeno. Sin embargo, las causas ecofisiológicas de esta respuesta deben ser analizadas en estudios específicos que comparen estas especies.

Los valores obtenidos de $\mathrm{IDR}_{\max }$ se encuentran dentro del rango reportado para diversas especies de coníferas y latifoliadas (Long 1985, Dean y Baldwin 1993, Wittwer et al. 1997, Río et al. 2001, Woodall et al. 2005). Sin embargo, para coigüe este valor es algo elevado. Chauchard et al. (1999) reportaron un $\mathrm{IDR}_{\max }$ para rodales mixtos de Nothofagus spp., el que lamentablemente no es comparable con estos resultados porque está basado en un diámetro de referencia de $30 \mathrm{~cm}$.

Para los diagramas se pueden definir diversas curvas o líneas de referencia, las cuales son clave para planificar intervenciones silvícolas y definir esquemas de manejo. La línea A corresponde a la curva de máxima ocupación del sitio o densidad (línea del 100\% de los diagramas) e identifica al estado de máxima competencia (Nowak 2002). Los rodales existentes sobre esta línea están en un estado de "sobredensidad" y deberían ser situaciones poco frecuentes.

La línea B corresponde al límite inferior para el inicio del autorraleo; es aquí cuando comienza a ocurrir mortalidad producto de la competencia por recursos (Long 1985). Esta línea además representa el nivel donde se está realizando una completa ocupación del sitio (Rogers 1983), y por ende es donde se maximiza el crecimiento bruto del rodal (Long 1985, Nyland 2002). Esta línea idealmente debería ser obtenida a partir de experimentos de raleo en parcelas permanentes, registrándose el momento cuando se empieza a producir mortalidad inducida por la competencia. Sin embargo, diversos estudios definen este nivel en un rango de 50 a $60 \%$ de la densidad máxima (Rogers 1983, Long 1985, Dean y Jokela 1992, Wittwer et al. 1998). Para el presente estudio, se optó por usar un nivel del $60 \%$.

La línea $\mathrm{C}$ corresponde al límite inferior de ocupación del sitio, y muchas veces corresponde al momento en que se produce el cierre de copas y se empieza a producir competencia entre individuos por los recursos (Dean y Baldwin 1993). Sin embargo, a diferencia de la línea B, esta competencia no induce una mortalidad inmediata. Gingrich (1967) obtiene esta curva usando una relación del área máxima de copa, la que luego es usada para determinar el número máximo de árboles de crecimiento libre que un rodal podría tolerar. Chauchard et al. (2001) ajustan una relación de área máxima de copa para árboles de roble, raulí y coigüe con diámetros entre 5 y $100 \mathrm{~cm}$ creciendo 
en Argentina. Esta curva, al ser comparada con la línea A obtenida por este estudio, corresponde a niveles de entre $16 \%$ y $19 \%$ de la densidad máxima, valores considerados bajos y que pueden deberse a los diferentes rangos de diámetros cuadráticos usados entre ambos estudios. Ante la falta de antecedentes de copa es posible definir el nivel como una proporción de la densidad máxima. Diversos autores han usado valores entre $25 \%$ y $35 \%$ para esta línea (Dean y Jokela 1992, Dean y Baldwin 1993, Wittwer et al. 1998). Para las composiciones estudiadas se optó por definir la línea $\mathrm{C}$ a un nivel intermedio del $30 \%$.

Finalmente, es posible definir la línea D, la cual es usada para determinar el nivel mínimo de densidad que un rodal puede tener y aún presentar la capacidad potencial de desarrollarse para constituir un rodal de densidad completa (Nowak 2002). No existen antecedentes disponibles para la determinación de esta línea, sin embargo, en relación con la línea A determinada por este estudio, el número mínimo de árboles a dejar recomendado por las normas de manejo de estos renovales (Lara et al. 1998) corresponde a niveles promedio de $17 \%$ y $22 \%$ para los subtipos roble-raulí y coigüe, respectivamente (cuadro 3). Se optó por definir esta línea en un nivel de un $20 \%$, el cual además corresponde a valores levemente superiores a los obtenidos usando el modelo de área máxima de copa de Chauchard et al. (2001) y, por lo tanto, permite asegurar una cobertura mínima del suelo.
En resumen, para el uso de estos diagramas se sugieren las siguientes líneas de referencia para las especies estudiadas: 100\% (línea A o de densidad máxima), 60\% (línea B o de inicio del autorraleo), 30\% (línea C o límite inferior de ocupación del sitio) y 20\% (línea D o nivel mínimo de densidad).

La planificación de los raleos idealmente debería tener por objetivo mantener la densidad del rodal entre las líneas $\mathrm{B}$ y $\mathrm{C}$, esto porque la línea $\mathrm{B}$ maximiza el crecimiento volumétrico del rodal y la línea $\mathrm{C}$ maximiza el crecimiento individual (Long 1985, Newton 1997). Dependiendo del producto final deseado se debería favorecer un nivel sobre otro. Por ejemplo, para la producción de trozas de madera aserrada es recomendable mantener las densidades cercanas a la línea C. Otros niveles pueden también ser de interés. Densidades menores al $30 \%$ favorecen el desarrollo del sotobosque y la regeneración natural, y niveles superiores al $60 \%$ permiten mantener un mejor control de especies arbustivas (Rogers 1983). Por otro lado, Nyland (2002) sostiene que niveles de hasta $80 \%$ podrían ser justificables ya que en estos casos la mortalidad se concentra en individuos suprimidos o intermedios, los que además podrían ser más apropiados para rodales dominados por especies tolerantes a la sombra. Por otro lado, que un renoval presente una densidad menor al $20 \%$ puede ser un indicador de que es necesario enriquecer el rodal con plantas de vivero.

Cuadro 3. Comparación de densidades para composición raulí-mixto y coigüe. DC: diámetro cuadrático, NHA: número de árboles por hectárea.

Density comparisons for raulí-mixed and coigüe compositions. DC: quadratic diameter, NHA: trees per hectare.

\begin{tabular}{|c|c|c|c|c|c|c|c|}
\hline \multirow{2}{*}{$\begin{array}{c}\mathrm{DC} \\
(\mathrm{cm})\end{array}$} & \multicolumn{4}{|c|}{ NHA (árboles / hectárea) } & \multicolumn{3}{|c|}{ Proporciones $(\%)$} \\
\hline & Máxima1 & Referencia ${ }^{2}$ & Mínima ${ }^{3}$ & Remanente $^{4}$ & Referencia & Mínima & Remanente \\
\hline \multicolumn{8}{|c|}{ Raulí mixto } \\
\hline 5 & 18.745 & 5.423 & 2.820 & 1.842 & 29 & 15 & 10 \\
\hline 10 & 7.048 & 3.528 & 1.842 & 1.203 & 50 & 26 & 17 \\
\hline 15 & 3.977 & 2.295 & 1.203 & 786 & 58 & 30 & 20 \\
\hline 20 & 2.650 & 1.493 & 786 & 513 & 56 & 30 & 19 \\
\hline 25 & 1.934 & 971 & 513 & 332 & 50 & 27 & 17 \\
\hline 30 & 1.495 & 632 & 332 & 250 & 42 & 22 & 17 \\
\hline 35 & 1.203 & 411 & - & - & 34 & - & - \\
\hline \multicolumn{8}{|c|}{ Coigüe } \\
\hline 5 & 21.698 & 6.339 & 4.161 & 2.913 & 29 & 19 & 13 \\
\hline 10 & 8.158 & 4.125 & 2.708 & 1.896 & 51 & 33 & 23 \\
\hline 15 & 4.604 & 2.685 & 1.763 & 1.234 & 58 & 38 & 27 \\
\hline 20 & 3.068 & 1.747 & 1.147 & 803 & 57 & 37 & 26 \\
\hline 25 & 2.239 & 1.137 & 747 & 523 & 51 & 33 & 23 \\
\hline 30 & 1.731 & 740 & 482 & 337 & 43 & 28 & 19 \\
\hline 35 & 1.393 & 482 & - & - & 35 & - & - \\
\hline
\end{tabular}

${ }_{1}^{1}$ Este estudio (FONDEF D97I1065), ${ }^{2}$ Puente et al. (1979), 3,4 Lara et al. (1998) 
El uso práctico de los diagramas de densidad es relativamente simple. Por ejemplo, un rodal de raulí que en su estado inicial tiene una densidad de 3.000 árboles/ha, $60 \mathrm{~m}$ /ha de área basal y un diámetro cuadrático de 16 $\mathrm{cm}$, de acuerdo a la figura 3 se encuentra a un $76 \%$ de la densidad máxima del sitio. Si los objetivos de manejo se establecen a fin de bajar la densidad hasta un $40 \%$, se sigue la línea de los $16 \mathrm{~cm}$ hasta llegar a la curva del $40 \%$, el que corresponde a 1.890 árboles/ha con 38 $\mathrm{m}^{2} /$ ha de área basal y, por lo tanto, se deberían extraer 22 $\mathrm{m}^{2} / \mathrm{ha}$, es decir, una intensidad de raleo del $37 \%$. En este caso, el diámetro cuadrático pre- y posraleo se mantiene constante, por ejemplo, con un raleo que afecta todas las clases diamétricas por igual. Este rodal se podría dejar crecer hasta que alcance la línea B, en donde se aplica otro raleo para volver a llegar a una densidad cercana al $40 \%$ y luego se deja crecer hasta alcanzar la edad de rotación, o algún diámetro objetivo. Es importante hacer notar que las normas de manejo de renovales permiten una extracción máxima en términos de área basal de un $35 \%$ para coigüe y un $40 \%$ para las otras composiciones (Lara et al. 1998). Sin embargo, en algunos casos el uso de estos diagramas podría indicar que raleos con extracciones mayores son posibles. En todos los casos, la estabilidad de los árboles remanentes debe siempre ser evaluado considerando otros aspectos tales como la relación altura-diámetro y la magnitud y dirección de los vientos dominantes.

El período y frecuencia de las intervenciones dependerá de las tasas de crecimiento, las que a su vez se ven afectadas por la productividad y edad del rodal. Esta última debe ser considerada cuidadosamente, dado que ella influye fuertemente en la capacidad de respuesta de los individuos. Así, en general, el período entre raleos será menor en rodales más productivos y jóvenes, pero también dependerá del producto final objetivo. En este sentido, para una adecuada planificación de las intervenciones futuras, el uso de estos diagramas puede ser apoyado con curvas de altura dominante y modelos de rendimiento o crecimiento.

Es importante destacar que los diagramas presentados en este estudio se basan en mediciones de parcelas de todos los árboles que presentan un DAP igual o superior a $5 \mathrm{~cm}$ de cualquier especie. Por otro lado, se asume que las diferencias de productividad y edad que se presenten en distintos rodales no afecten considerablemente los resultados. Sin embargo, bajo condiciones extremas es probable que se produzcan desviaciones de magnitud. En este sentido, los diagramas presentados en la figura 3 deben ser utilizados respetando los rangos de las observaciones utilizados para su construcción (cuadro 2). El uso en otras situaciones corresponde a una extrapolación, y debe ser evaluado con cautela.

\section{AGRADECIMIENTOS}

La mayoría de los datos de terreno para este estudio fueron obtenidos durante la ejecución del proyecto FONDEF D9711065 "Software de Planificación de Actividades en Renovales de Roble, Raulí y Coigüe en la IX y X Región" de la Universidad Austral de Chile, el que fue financiado por CONICYT junto a empresas e instituciones asociadas. Se agradece el valioso apoyo recibido de Paulo Moreno, Marcelo Farías y varios brigadistas de terreno, y de un árbitro anónimo por su dedicada labor.

\section{REFERENCIAS}

Chauchard L, R Sbrancia, M González, L Maresca, A Rabino. 1999. Aplicación de leyes fundamentales de la densidad a bosques de Nothofagus: I. Regla de los $-3 / 2$ o ley del autorraleo. Bosque 20:79-94.

Chauchard L, R Sbrancia, M González, L Maresca, A Rabino, M Mazzuchelli. 2001. Aplicación de leyes fundamentales de la densidad a bosques de Nothofagus: II. Línea de inicio de competencia y diagramas de manejo de la densidad. Bosque 22:3-10.

CONAF (Corporación Nacional Forestal, CL), CONAMA (Comisión Nacional del Medio Ambiente, CL), BIRF (Banco Internacional de Reconstrucción y Fomento, USA), Universidad Austral de Chile, Pontificia Universidad Católica de Chile, Universidad Católica de Temuco. 1999. Proyecto catastro y evaluación de los recursos vegetacionales nativos de Chile. Santiago, Chile. 87 p.

Day K. 1998. Stocking standards for uneven-aged interior douglasfir. In Vyse A, C Hollstedt, D Huggard eds. Managing the dry Douglas-fir forests of the southern interior: Workshop Proceedings. Victoria, Canada. B.C. Min. For. p. 37-52.

Dean TJ, VC Baldwin. 1993. Using a density-management diagram to develop thinning schedules for loblolly pine plantations. Res. Pap. SO-275. New Orleans, LA: U.S. Department of Agriculture, Forest Service, Southern Forest Experiment Station. 7 p.

Dean TJ, EJ Jokela. 1992. A density-management diagram for slash pine plantations in the lower coastal plain. South. J. Appl. For. 16:178-185.

Donoso P, C Donoso, V Sandoval. 1993. Proposición de zonas de crecimiento de renovales de Roble (Nothofagus obliqua) y Raulí (Nothofagus alpina) en su rango de distribución natural. Bosque 14:37-55.

Donoso C, L Gallo, P Donoso, MM Azpilicueta. 2004. Variación de Nothofagus obliqua (Mirb.) Oerst. (Roble, Coyán, Hualle o Pellín). In Donoso C, A Premoli, L Gallo, R Ipinza eds. Variación Intraespecífica en las especies arbóreas de los bosques templados de Chile y Argentina, Editorial Universitaria. Santiago, Chile. p. 79-113.

Echeverría C, A Lara. 2004. Growth patterns of secondary Nothofagus obliqua-N. alpina forests in southern Chile. For. Ecol. Manage. 195:29-43.

Gingrich SF. 1967. Measuring and evaluating stocking and stand density in upland hardwood forests in the Central States. For. Sci. 13:38-58. 
Grosse H, I Quiroz. 1998. Silvicultura de los Bosques de Segundo Crecimiento de Roble, Raulí y Coigüe en la Región CentroSur de Chile. In Donoso C, A Lara eds. Silvicultura de los Bosques Nativos de Chile, Editorial Universitaria. Santiago, Chile. p. 95-128.

Lara A, C Donoso, P Donoso, P Núñez, A Cavieres. 1998. Normas de manejo para raleo de renovales del tipo forestal roble-raulí-coigüe. In Donoso C, A Lara eds. Silvicultura de los Bosques Nativos de Chile, Editorial Universitaria. Santiago, Chile. p. 129-144.

Long JN. 1985. A practical approach to density management. For. Chron. 61:23-27.

Martínez A. 1998. Silvicultura práctica en renovales puros y mixtos, y bosques remanentes originales del tipo forestal Roble-Raulí-Coigüe. In Donoso C, A Lara eds. Silvicultura de los Bosques Nativos de Chile, Editorial Universitaria. Santiago, Chile. p. 145-175.

Newton PF. 1997. Stand density management diagrams: reviews of their development and utility in stand-level management planning. For. Ecol. Manage. 98:251-265.

Nowak C. 2002. Stocking charts as a way to organize stand complexity. The New York Forester 58(4):5-6.

Nyland R. 2002. Silviculture: concepts and applications. Second edition. McGraw-Hill, New York, USA. 704 p.

Puente M, C Donoso, R Peñaloza, E Morales. 1979. Estudio de raleos y otras técnicas para el manejo de renovales de raulí y roble. Etapa I: Identificación y caracterización de renovales de raulí y roble. Informe de convenio $\mathrm{N}^{\circ} 5$. Proyecto CONAF/PNUD/FAO-CHI, 128 p.

Reineke LH. 1933. Perfecting a stand-density index for even-aged forests. J. Agric. Res. 46:627-638.
Roach BA. 1977. A stocking guide for Allegheny hardwoods and its use in controlling intermediate cuttings. Res. Pap. NE-373. Upper Darby, PA: U.S. Department of Agriculture, Forest Service, Northeastern Forest Experiment Station. 30 p.

Rodríguez C. 1993. Estimación de la mortalidad natural en los renovales de roble y raulí entre 20 y 50 años de edad. Tesis Ingeniero Forestal. Valdivia, Chile. Universidad Austral de Chile. 84 p.

Río M del, G Montero, F Bravo. 2001. Analysis of diameter-density relationship and self-thinning in non-thinned even-aged scots pine stands. For. Ecol. Manage. 142:79-87.

Rogers R. 1983. Guides for thinning shortleaf pine. In Proceedings of the second biennial southern silvicultural research conference. Atlanta, GA, USA. U.S. Department of Agriculture, Forest Service. p. 217-225.

SAS Institute. 1999. SAS Version 8.0. SAS Institute, Cary, North Carolina, USA.

Wittwer R, T Lynch, M Huebschnann. 1997. Stand density index for shortleaf pine (Pinus echinata Mill.) natural stands. In Proceedings of the ninth biennial southern silvicultural research conference. Asheville, NC, U.S.A. U.S. Department of Agriculture, Forest Service. p. 590-596.

Woodall CW, PD Miles, JS Vissage. 2005. Determining maximum stand density index in mixed species stands for estrategic-scale stocking assessments. For. Ecol. Manage. 216: 367-377.

Zeide B. 1987. Analysis of the $3 / 2$ power law of self-thinning. For. Sci. 33: 517-537.

Zeide B. 1995. A relationship between size of trees and their number. For. Ecol. Manage. 72: 265-272. 